\title{
Conceptualization of the Human-Machine Symbiosis A Literature Review
}

\author{
Alina Gerber \\ University of Cologne \\ gerber@wim.uni- \\ koeln.de
}

\author{
Patrick Derckx \\ University of Cologne \\ derckx@wim.uni- \\ koeln.de
}

\author{
Daniel A. Döppner \\ University of Cologne \\ doeppner@wim.uni- \\ koeln.de
}

\author{
Detlef Schoder \\ University of Cologne \\ schoder@wim.uni- \\ koeln.de
}

\begin{abstract}
The vision of a symbiotic partnership between humans and machines has existed since the 1960s. With this paper we provide the first conceptualization of the human-machine symbiosis (HMS) and make three important contributions: we present the fundamentals of HMS by focusing on objectives, requirements, and boundaries; we propose a framework for the design of HMS; and we review HMS research and, specifically, what the literature says with respect to whether HMS has already been achieved.
\end{abstract}

\section{Introduction}

The continuing, and relentless, development of information technology (IT) has been opening up new opportunities for humankind for decades. Things that for previous generations could only imagine have become part of everyday life.

Back in 1960, Licklider formulated his vision of a "Man-Computer Symbiosis" in which he calls for a close cooperative relationship between humans and machines that would be capable of thinking in ways no human brain had ever done and process data that machines of the time could not handle [27]. His call was far ahead of its time, particularly given that computers of the time were hardly user-friendly, and advances such as the mouse and graphical user interfaces had not yet been developed.

Today, innovations in the field of artificial intelligence (AI) have changed the world significantly. AI now helps computers better understand situations and react to them. AI demonstrates the ability of IT to extend the possibilities of what can be automated, and how IT can team together with humans on complex problem solutions, such as organizational decision making [22]. This idea of joint problem solving is increasingly being taken up in both theory and practice as we move towards a "race with the machines" [10]. This requires us to rethink how information systems are designed, built, and deployed. To achieve this, it is increasingly important to understand teaming humans and machines together as a symbiotic relationship.

Abbass et al. describe the literature on humanmachine symbiosis (HMS) in a recently publication as "very rich and diverse" [1]. To the best of our knowledge, however, there is no foundational work that goes beyond Licklider's vision to consolidate the literature and provide a conceptualization of HMS. Thus, the time has come to build a vocabulary for future research. A first and essential step is to review and structure the existing research in this rich and diverse field. Doing so will help answer the following research question:

RQ: What should a conceptualization of the human-machine symbiosis look like?

In this paper, we provide a first conceptualization of the HMS and make three important contributions: we present the fundamentals of HMS by focusing on objectives, requirements, and boundaries; we propose a framework for the design of HMS; and we review HMS research and, specifically, what the literature says with respect to whether HMS has already been achieved.

The rest of this paper is structured as follows. We next provide, in section 2, background on the terms "symbiosis" and "human-machine symbiosis." Section 3 introduces the methodological approach for our literature review. In the subsequent sections, we present the results of the research, beginning with the conceptualization in section 4 , where we address the objectives, requirements, and boundaries. In section 5 , we show how HMS systems are designed and make an approach for a design framework. In section 6, we present the current status of HMS and offer an outlook for the future development of HMS. We interpret and discuss the results at the end of each of these three sections. Finally, in section 7, we summarize our work, discuss the limitations of our study, and offer recommendations for further research. 


\section{Background}

\subsection{Symbiosis}

Botanist Anton de Bary introduced the term symbiosis in 1879 to describe any type of coexistence of different organisms [5]. Since then, symbiosis has been adopted by other sciences, such as psychology [28]. Licklider made use of the definition from biology and was the first to extend the term to non-biological artifacts [27, 40]. Several authors emphasize that Licklider' made metamorphic use of the term symbiosis, since computers are not living entities [17, 18]. In the literature, however, the term symbiosis is no longer restricted to organisms, but has been extended to non-living entities, including machines, as possible actors in symbiotic relationships $[9,11]$. Hence, machines can be part of a symbiotic relationship. Humans and machines are, therefore, referred to as actors rather than organisms in the sections that follow.

De Bary suggests that not every form of symbiosis can be treated equally because they have different objectives within the relationship. He specifically mentions two: parasitism, a relationship in which only one actor (the parasite) benefits from living together; and mutualism, a symbiotic relationship in which both actors benefit as partners. Symbiosis is often referred to in that mutual form $[21,36]$. Didakis points out that this interpretation also applies in connection with HMS [12]. Griffith even emphasizes explicitly that the term symbiosis can be used only in the case of coequality of the partners [18].

For the evaluation in this literature study, the term symbiosis is equated with the definition of mutual symbiosis. This assumption is relevant because it defines symbiosis as pursuing a common objective.

\subsection{Human-Machine Symbiosis}

From a morphological perspective, the term human-machine symbiosis further develops Licklider's terminology of a Man-Computer Symbiosis. and is a compound consisting of three words. For the elaboration of the meaning of the term, it is divided into its three components [6]:

- Human: we use the gender-neutral term more common today [18].

- Machine: defined as "an apparatus, consisting of a number of interrelated parts, constructed to perform a task" [50], which thus encompasses not only computers and software but also allows for including other technological developments such as robots, smartphones and virtual reality glasses [29].
- Symbiosis: the coexistence of actors of different kinds for mutual benefit.

Together the three words form the term humanmachine symbiosis. Accordingly, HMS is the coexistence of the human and machine actors for mutual benefit. This covers all aspects of Licklider's Man-Computer Symbiosis, but is also adapted to today's technologies and terminologies.

HMS distinguishes itself from other humanmachine relationships precisely because of the partnership and mutual benefit. In the symbiosis, the human and the machine benefit primarily from the fact that "both parties [become] smarter over time" [24]. Whereas the machine would not even exist without the human being, and needs continual input to function properly [13], the human has in many ways become dependent on the machine for its efficiency, for example, in calculations [15, 24].

\section{Method}

In this study, we conducted a concept-oriented and systematic literature search based on Okoli and Schabram as well as Webster and Watson $[32,46]$. The search process consists of two steps. In both, we screened the titles and abstracts of articles we found to identify the relevant ones (literature filtering). The criterion for relevance was that at least one of the following must be addressed in the paper: (1) HMS itself; (2) the objectives of HMS; and/or (3) the design of HMS. As no uniform conceptualization of HMS yet exists, we included only those papers that explicitly use the term symbiosis. In addition, we limited our selection to peer-reviewed publications.

The first step involved a forward search from Licklider's Man-Computer Symbiosis. The article was cited 1987 times. After the literature filtering, we identified 16 titles (including Licklider) as relevant. In a second step, six databases (ProQuest, AISeL, ACM DL, EBSCOhost, IEEE Xplore, ScienceDirect) are searched for "(man OR human) AND (machine OR computer OR technology) AND symbio*," resulting in 688 articles found. After the literature filtering, this was reduced to 13 publications. We then found another 7 titles by forward and backward search. In the end, our search yielded 36 publications.

We structured the relevant papers with the help of a concept matrix. To identify the concepts, we applied the principles of Grounded Theory as proposed by Wolfswinkel et al. (2013) [47]. The individual papers were successively screened and open coding was applied. Open coding facilitates the identification, naming, and summary of concepts through repeated analysis of the texts. The content of a concept is 
determined by the so-called properties. We identified six concepts: objectives, requirements, boundaries, design, current state, and future of HMS. For greater clarity, we grouped the concepts into categories that capture groups of concepts. These can be either previously studied concepts or new concepts that emerge as logical when thinking through the grouping of the concepts identified.

Employing this method, we developed three categories to describe what is included by the authors of the papers:

- Fundamentals: includes as concepts the objectives, requirements, and boundaries of HMS.

- Design: includes guidelines for the development of HMS.

- State: addresses the state of HMS. A distinction is made between discussing the current state and projecting a future state.

\section{Fundamentals of HMS}

\subsection{Objectives}

HMS has a mutualistic character, therefore mutual benefits from the relationship can be assumed. This is shown in set of objectives discussed below.

One of these objectives is to create an effective system, the achievement of which requires that human and machine are not considered individually but rather as a unit in the form of a system [23]. The system becomes effective by combining the strengths of both actors to achieve what was previously unattainable for the individual $[12,13,20]$.

This effectiveness stems further from the fact both human and machine are optimized as a whole towards a common goal [20,33]. Cooperation is the focus of an effective system, aimed at optimally bundling all capabilities [38] in order to implement a perfect, dynamic division of tasks [33]. Overcoming human restrictions is another focus [15, 19]. The machine improves and expands human capabilities [29]. Furthermore, the technology supports and HMS creates new possibilities and approaches for problem solving $[4,19]$.

The ideal use of resources is another objective [33], with the focus on the temporal aspect. Maier et al. argue that HMS can reduce the time needed to solve problems [29]. Improving human capabilities optimizes processing time and goals can be reached more quickly [31].

Licklider formulated the effective system and the time factor as essential objectives of Man-Computer Symbiosis, postulating that the symbiosis would solve impossible and unimaginable problems and produce time-saving results [27]. The literature concurs.

A third main objective of HMS is to create humanlike communication and interaction, which is crucial to enable actors to accept each other as equal partners [39]. Humanlike communication includes both verbal (spoken and written language) and non-verbal communication (gestures, facial expressions, emotions) $[40,44]$. The machine has to understand the different aspects of human communication and use them in an exchange with the human.

A final aspect the literature highlights is that HMS goes beyond automation [24] to provide a solution when automation alone is not sufficient [34]. As this appears in only three publications, we do not regard it to a main HMS objective.

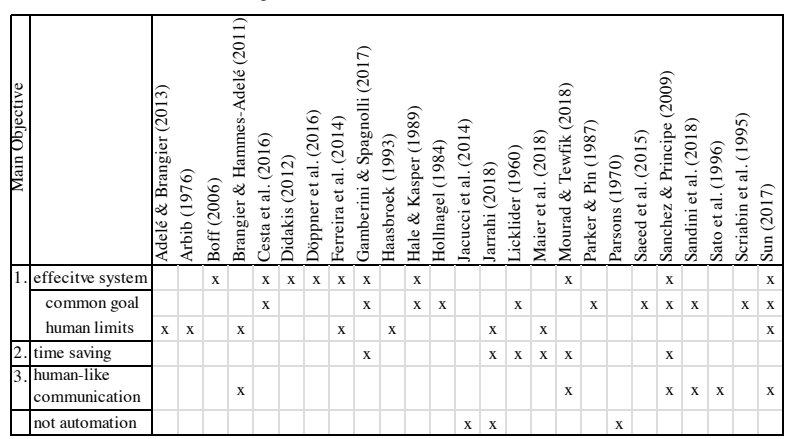

Table 1: Objectives of HMS

In his 1960 work, Lickider described the achieving of previously unattainable goals by working together in an effective system, saving time, and employing human-like communication as resulting in the optimal efficiency of the system of human and machine. Table 1 summarizes which papers from our literature search address the objectives just discussed.

\subsection{Requirements}

As a consequence of the different priorities for an effective system (section 4.1), the requirements of HMS also differ. A distinction can be made between requirements for the actors, the system and the machine.

Different arguments can be found for the number of actors involved in HMS. Even if not made explicit in the papers, in most cases we are talking about one human and one machine. Three papers specifically highlight this $1: 1$ relationship $[11,39,42]$. Xu et al., however, postulate that a limit of only two actors is not suitable for grid computing [48]. Lessiter et al. argue that symbiosis has no limit in the number of actors [26]. As there is no definite consensus, we assume a 1:1 relationship.

There is consensus, though, regarding the role of the machine in the relationship. The machine is 
regarded as a partner in an equal position [2, 24]. This is reflected in a balance of power: an optimal HMS operates without superior control by either side [40]. This suggests that technology supports humans but should not be seen by people as a mere means to an end. Petriu et al. formulate this as a constant change in the roles of the two actors, alternating between partner and assistant [35].

How the human perceives HMS is important. It is the interaction as partners that generates the effectiveness of HMS [24]. To employ the full potential of HMS, there must be more than simple acceptance of the machine from the human side [2]. Having the human see the machine as a partner is made possible by creating the relationship instinctively and naturally [8]. The "human friendliness" that is thus created [41], which enables not only user-friendly handling but also instinctive handling of the machine, is an essential characteristic of symbiosis. HMS is, therefore, characterized by a relationship with a natural character.

There are requirements for the system in addition to those for the relationship. Twelve papers emphasize that the system in HMS must be dynamic [2, 13]. In concrete terms, this means that both humans and machines must be able to adapt to new situations in real time [39]. All actors must play several clearly defined roles, and must also be able to learn in any context $[21,38]$. The latter presupposes that all actors are regarded as intelligent [4, 21, 45]. It is further emphasized that machines must not only act based on rules, but must also possess and master creativity and intuitive action [24].

The requirement for a dynamic system necessitates the optimal use of resources. For example, there must be a clear division of tasks within the HMS to enable the creation of timely solutions $[19,25]$. The focus must be on the effectiveness of the entire system, not on the individual [8]. Storage capacity matters as well. While the literature does not specify orders of magnitude, there is an emphasis that storage capacity must be sufficient large so as not to be a limit for the system [8].

Finally, the literature establishes three concrete requirements for the machine. The first is space awareness. Although a large part of human-machine interaction takes place in a virtual space, the human is always in the physical world [41]. The machine must have an awareness of the human environment so it can react to changes in that environment [40]. Furthermore, if the machine (e.g., a robot) is to interact with humans in the real world, it must be mobile and able to move in the human environment $[35,40]$.

The second requirement is representation. In addition to awareness of the environment, the machine must also have an understanding of the actors and the system [39]. This requires that the machine possess a model or profile of the human, the machine itself, and the system in which data can be collected [23, 44]. This will allow the machine to respond optimally to all interactions, and to gain new insights [11,31]. This representation, as the literature emphasizes in particular, can be successful only if there is constant transparency [23, 44], that is, if the machine discloses what data it collects and possesses and humans are not allowed to withhold any information [23]. In this context, security is a decisive factor [38]. Data security is the condition for transparency within the system.

Communication, the third requirement, is a decisive quality factor in HMS [14]. When Licklider formulated the Man-Computer Symbiosis in 1960, there was not even a computer mouse to use for input [40]. Today, input via keyboard, mouse, touch screen, and voice are all easy, and output is via user-friendly GUI [23]. Nevertheless, this form of communication is not sufficient for complete HMS [21, 30], since human communication is not only verbal and gesture-rich, but is also context-dependent and emotionally shaped [21, 45]. The machine must, therefore, understand all these facets of human communication if it is to interact with people smoothly and effortlessly. This places high demands on communication in an HMS, since

\begin{tabular}{|c|c|c|c|c|c|c|c|c|c|c|c|c|c|c|c|c|c|c|c|c|c|c|c|c|c|c|c|c|c|c|c|c|c|}
\hline & & 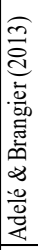 & 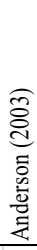 & 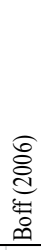 & 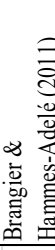 & & 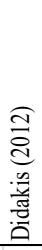 & 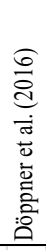 & 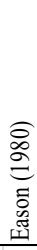 & 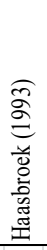 & 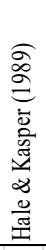 & 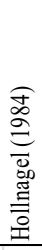 & 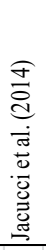 & 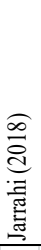 & 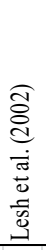 & 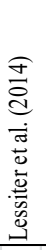 & 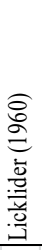 & 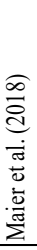 & 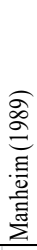 & 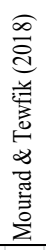 & 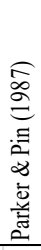 & 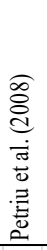 & 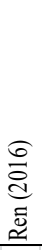 & 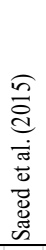 & 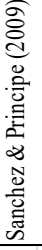 & 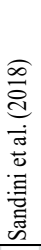 & 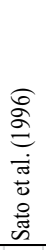 & 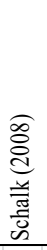 & 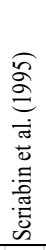 & 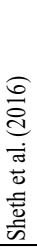 & 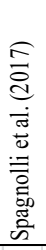 & 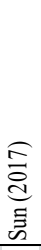 & 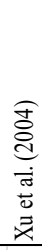 \\
\hline \multirow{3}{*}{ 苞 } & \multirow{3}{*}{$\begin{array}{l}\text { number of actors } \\
\text { role of the machine } \\
\text { natural character }\end{array}$} & & & & & $\mathrm{x}$ & & & & & & & & & & $\mathrm{x}$ & & & & & & & & & $\mathrm{x}$ & & & & $\mathrm{x}$ & & & & $\mathrm{x}$ \\
\hline & & $x$ & & & $\mathrm{x}$ & & & & & & & $x$ & & $x$ & & & $x$ & & & & & $x$ & & & $\mathrm{x}$ & $\mathrm{x}$ & & & $\mathrm{x}$ & & & $\mathrm{x}$ & \\
\hline & & $x$ & $\mathrm{x}$ & $\mathrm{x}$ & & & & & & & $\mathrm{x}$ & & $\mathrm{x}$ & $\mathrm{x}$ & & $\mathrm{x}$ & $x$ & & & $\mathrm{x}$ & & & & & $\mathrm{x}$ & & $\mathrm{x}$ & & & & & & \\
\hline \multirow{2}{*}{ 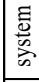 } & \multirow{2}{*}{$\begin{array}{l}\text { dynamic } \\
\text { optimal use of resources }\end{array}$} & $x$ & & & $\mathrm{x}$ & $\mathrm{x}$ & & $\mathrm{x}$ & $\mathrm{x}$ & & & & & $x$ & & & & & $\mathrm{x}$ & $x$ & $\mathrm{x}$ & $\mathrm{x}$ & & $\mathrm{x}$ & $\mathrm{x}$ & $\mathrm{x}$ & & & $x$ & $\mathrm{x}$ & & $\mathrm{x}$ & \\
\hline & & & & $\mathrm{x}$ & & $\mathrm{x}$ & & $\mathrm{x}$ & & $\mathrm{x}$ & & & & & $\mathrm{x}$ & & & & & & & & & & $\mathrm{x}$ & & & & & & $\mathrm{x}$ & & \\
\hline \multirow{3}{*}{ 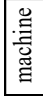 } & \multirow{3}{*}{$\begin{array}{l}\text { space awareness } \\
\text { representation } \\
\text { natural communication }\end{array}$} & & & & & $\mathrm{x}$ & & & & & & & & & & & & $\mathrm{x}$ & & & & $\mathrm{x}$ & & & $\mathrm{x}$ & $\mathrm{x}$ & & & & & & & \\
\hline & & & & & $\mathrm{x}$ & $\mathrm{x}$ & & & & & & & $x$ & $x$ & $x$ & & & $\mathrm{x}$ & & & & & $\mathrm{x}$ & $x$ & $x$ & $\mathrm{x}$ & & & & & & $x$ & $\mathrm{x}$ \\
\hline & & & & $\mathrm{x}$ & & & $\mathrm{x}$ & & $\mathrm{x}$ & $\mathrm{x}$ & & $\mathrm{x}$ & $\mathrm{x}$ & $\mathrm{x}$ & $\mathrm{x}$ & & & & $\mathrm{x}$ & & & $\mathrm{x}$ & & & & $\mathrm{x}$ & $\mathrm{x}$ & $\mathrm{x}$ & & & $\mathrm{x}$ & $\mathrm{x}$ & \\
\hline
\end{tabular}

Table 2: Requirements of HMS 
misinterpretation represents a large potential for error.

Table 2 correlates the eight requirements to the papers in our literature review. Each requirement is represented in enough papers to suggest that all of them must be considered when designing HMS. However, the diversity of focal points suggests that context matters. Space awareness and natural communication are particularly important for mobile machines, since they move in the physical world and interact with humans. A software program that calculates and provides information on life-support measures in medicine, for instance, depends more on correct representation with complete and up-to-date data.

\subsection{Boundaries}

The concept of boundaries is least represented in the literature; it appears in only eight of the papers in our search. Nevertheless, understanding boundaries is indispensable, as they determine the cases in which HMS cannot be achieved or is not the optimal solution.

The most frequently cited cause of failure in trying to achieve HMS is lack of trust in the machine. It must, therefore, be an aim for the human to understand the machine's behavior so that trust can be established [45]. A person can trust the machine only if that person knows how the machine works and arrives at its results [24]. Otherwise, HMS cannot be achieved.

However, symbiosis also holds great dangers because of its requirement for transparency and openness. Jacucci et al. argue that much personal and sensitive data are exchanged in HMS and that the risk of misuse is particularly high [23]. They conclude that for this reason HMS can be used only when data security is guaranteed.

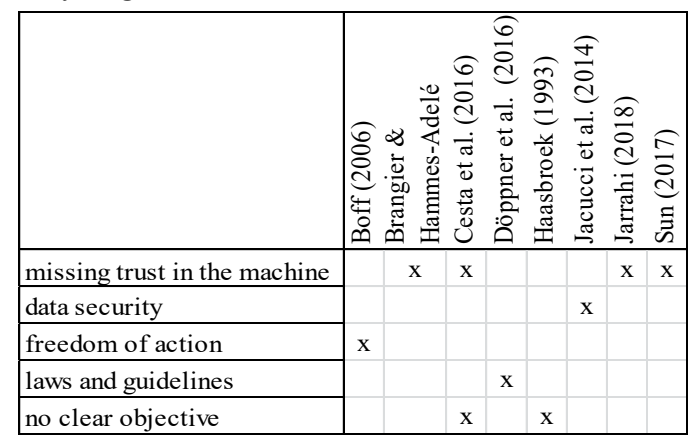

Table 3: Boundaries of HMS

In addition, HMS also potentially endangers the identity of the user [8]. Boff sees the danger of humans adapting to the system and losing their identity in the process [8]. He concludes that HMS is implementable only in circumstances where human are not forced to act against their will (freedom of action).
HMS can also reach its limits through its contextdependent use. Some areas always require human control due to laws and guidelines for higher security, for example [13]. This creates a power imbalance between the human and the machine that runs counter to the requirements of symbiosis.

The human factor is another reason for the failure of the system is the human factor. Section 4.1 explains that one objective of HMS is to achieve a common goal. However, it becomes problematic if the human does not have a precise idea of the objective [11,19].

As Table 3 shows, the literature on HMS boundaries reveals five failure criteria. The factors cover different aspects, but all of them show that HMS is not the optimal solution in each case. The limits can come from the context of the situation, ethical concerns and regulations, as well from the people themselves.

\subsection{Discussion}

The category fundamentals, which is addressed in all 36 publications, incorporates the differing perspectives that stem from different research areas and integrates them into a uniform understanding of HMS.

The discussion in the papers show that HMS is a goal-oriented relationship between human and machine that benefits both actors in a time-saving manner. The purpose of the relationship is to achieve a clearly defined and common goal that could not be realized individually. The advantage for the actors is the improvement of their own performance by overcoming restrictions (human) and gaining the ability to learn (machine). Although human and machine benefit individually from the relationship, the actors in a successful HMS are regarded as a single unit. Consequently, the overall system and not just the individuals must be constantly optimized, which requires that the system must be designed dynamically to adapt quickly to circumstances and ensure optimal use of resources. Within the system, the actors must be regarded as equal partners that interact intuitively. To ensure that, natural communication and transparent information management are essential components of HMS. HMS can therefore be implemented only in those cases in which the power symmetry is not restricted by, for example, external factors such as laws, data security and the lack of trust in the machine. The literature, however, says nothing about what information and data must be stored concretely. This aspect should be further explored in future research, taking into account that the design of the information needs depends on the HMS application area and that this varies in the different research areas. 


\section{Design of HMS}

As already discussed (section 4.2), symbiosis has different characteristics, and hence there is no uniform, concrete HMS design. Similarities, though, can be found at an abstract level. This section highlights the found concepts, as well as the actors (a), components (c), and relations (r) of our proposed framework.

HMS is typically considered part of humancentered design [37, 43], a process focused on user needs [37]. ISO 9241-210 defines the process as "based upon an explicit understanding of users, tasks, and environments," involving users "throughout design and development," and "driven and refined by usercentered evaluation" [49]. This definition should, therefore, be applied as a guideline for HMS.

The requirements for HMS suggest more concrete design specifications. One is regulated information management (c1). Jacucci et al. explain that tracking the environment is not sufficient to achieve HMS [23]. The machine also needs to capture human language and feelings [23]. In this way, it can perceive the environment (space awareness) and collect information about the state of the human and itself (representation). This goes hand in hand with the idea that data are collected constantly or at least periodically [17]. This information retrieval process takes place through information and data input by both actors (r1) $[23,38$, 39]. The models of the human (c11) and the machine (c12), which each represent the state of the actors, are extended by a context and a task model [39]. The context model meets the demand for space awareness, that is, the recording of the environment, with the addition of time and interaction data (c13) $[25,35]$. The task model, meanwhile, contains all information on the tasks and their process status (c14) [11, 25]. HMS must, therefore, be designed in such a way that information is available or can be obtained at any time by the actors (r2) $[44,45]$. The section above on requirements already elaborated that the machine must store the data needed for this purpose (r3) $[23,44]$.

Another requirement, adaptability for optimal resource use, must also be considered [25]. Five of the papers refer to Fitt's HABA (humans are better at) and MABA (machines are better at) list [16]. As section 4.1 discusses, however, it is important that the partners not only complement each other in their tasks, but function as a unit. That is achieved through task allocation [34], which is based on a third component besides the human (a) and the machine (a) [25] that we refer to as the task allocator (c2). This component is called by different names in the literature, but its purpose is always to coordinate the work dynamically, that is, to distribute the tasks between the actors $(\mathbf{r} 4)[11,14,33]$.

Optimal distribution depends on the situation [38]. The task allocator must therefore have access to the information management component so it can obtain the latest status of the actors and current tasks and update the task model if required (r5) $[11,38]$. The advantage of a task allocator lies in its dynamic aspect, which makes it possible to work quickly and save costs $[11,14,39]$.

Table 4 highlights these three aspects of HMS design. Human-centered design is the approach; information management and task allocation are the form of the design. Figure 1 integrates these into a

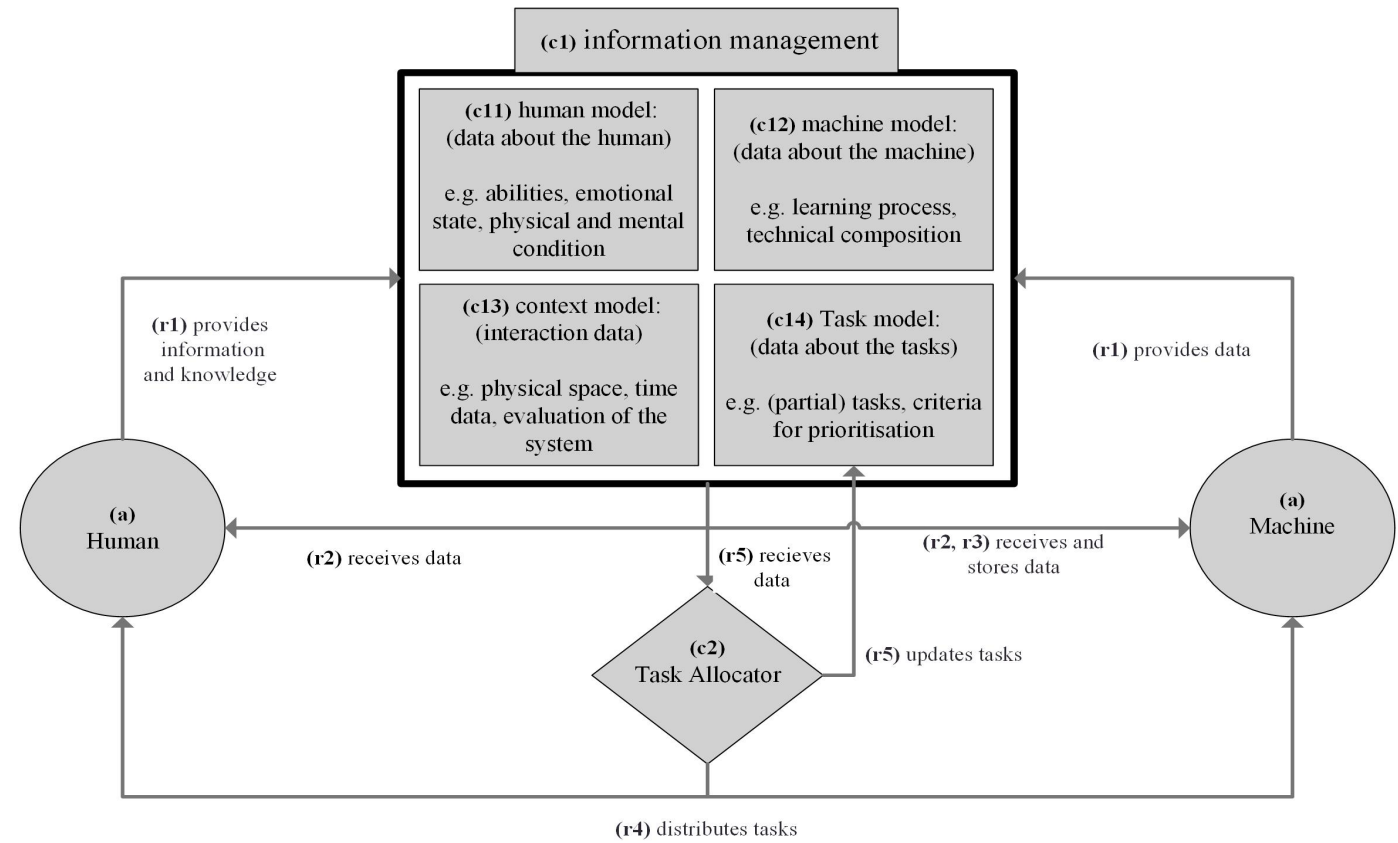

Figure 1: General Framework of a HMS System 
proposed framework for the design of an HMS system that comprises a system with two actors, the task allocator, and the information management. Information management consists of four submodels: the human and machine models contain relevant information on the respective actors; the context model contains all information about the interaction between the two; and the task model stores information on the tasks of the system and on the criteria for prioritizing those tasks.

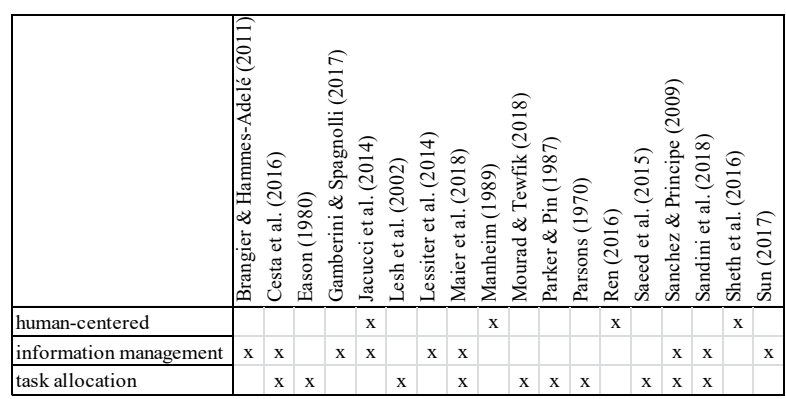

Table 4: Design of HMS

Information management data are stored by the machine and can be retrieved by human and machine at any time. The data for the models are provided by the two actors and are updated and adapted dynamically to ensure that they are current.

The task allocator, which distributes the tasks to the two actors, is another component of the system. It not only takes its data from the task model, but also includes data from all four submodels in the distribution process. The central outsourcing of distribution ensures that human and machine are regarded as one unit and that an optimal decision is made for the efficiency of the system.

\subsection{Discussion}

While the 18 relevant publications come from different research areas, common features can be found in the components they set out for the design of HMS. Even so, it is noteworthy that there is still no generic framework for the design of HMS in the papers. The framework we propose results from a consideration of all the papers and is therefore not limited to one field of application. Its value lies in the fact that it can be used as a basis for HMS design and can be extended and adapted according to a given situation.

\section{The State of HMS}

\subsection{The Current State of HMS}

In the course of comparing differences and similarities in the literature, we also reviewed authors' assessments of whether HMS has already been achieved. In this section, we consider only those publications that include a direct statement about the implementation status of HMS.

Six papers state that HMS has already been achieved [3, 23]. The authors argue that the development of new technologies automatically leads to new (symbiotic) relationships with machines [3]. In some cases, the papers go so far as to state that the human becomes dependent on the machine and loses certain abilities [9], such as in the case of the growing inability to remember phone number in the presence electronic equivalents of phone books [2]. Such dependence can be transformed into a partnership through proper design and handling [2].

In contrast, five papers argue that these sorts of examples do not yet correspond to $\operatorname{HMS}[15,40]$ because they are not the effective systems of HMS [35]. Thus, symbiosis is still in its infancy and requires further development [24].

All in all, as these contrasting positions show, there is no clear explanation of the current state of HMS in the literature. However, the year of publication is relevant. Those from the period 2000 to 2004 uniformly rank HMS as achieved, whereas those from 2005 on have a variety of opinions. The development of technologies may explain these differences. The earlier papers presumed that HMS had been achieved because a symbiotic system had been established with the (limited) technology of that time. From that point on, and especially with the introduction of Web 2.0, the capabilities of the technology expanded rapidly, opening up new and greater possibilities of HMS. The field of research may also explain some of the differences: computer scientist tend to fall in the camp of HMS achieved, whereas the other side consists mainly of engineers. This could reflect a view that symbiosis with computers or systems can already be achieved, but that it is not yet possible in the physical world with, for example, robots.

\subsection{The Future of HMS}

Beyond their assessments of the current state of HMS, the publications also offer an outlook for the future. Since cooperation in research is a key factor in the design of HMS, it is also relevant for the further development of HMS. It is needed to enable progress with respect to aspects that are still missing or are immature, such as dynamic learning and boundaries of HMS [20, 23].

HMS is also relevant with regard to the workplace of the future, particularly with respect to so-called Industry 4.0 and automation and the widespread discussion of potential job losses. Above all, the loss of "simple, routine" work and the prospect of a 
technological "singularity" are critical arguments against the symbiotic relationship between human and machine [21]. The papers, however, argue that optimal HMS is not to automate all processes, but that HMS acknowledges that humans possess abilities the machine cannot attain [31].

Finally, some papers do not see HMS as the final optimal design option for human-machine interaction. Boff and Sandini et al. explain that in the future the connection should be made not between human and machine but at a more biological level, such as braincomputer symbiosis [8, 40]. Boff goes on to explain that in the next generation of relationships, humans will adapt to the new system at the biological level until human and machine eventually merge [8].

Table 5 summarizes the outlook for HMS, presenting three essential aspects.

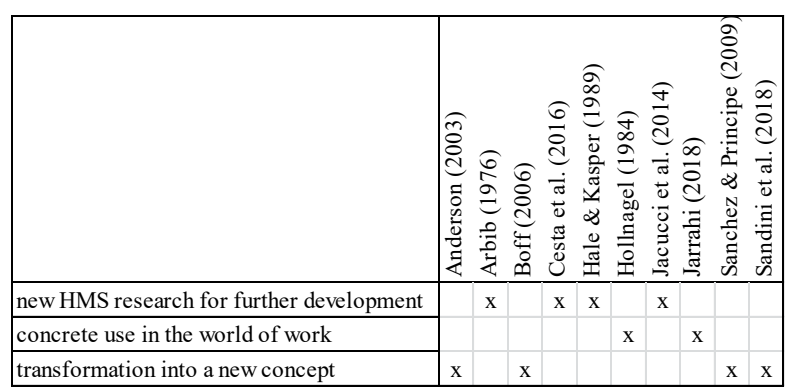

Table 5: The future of HMS

The investigation of the possible future of HMS does not provide a direct conclusion on the positioning of HMS in the current research literature, but supports the understanding of HMS. The authors' calls for further research and greater cooperation among the various fields confirms the interdisciplinary character of HMS.

Furthermore, explanations on the future development and use of HMS in the workplace highlight that symbiotic relationships will be a critical component in human-machine interaction. This shows that HMS is not only an important research topic, but also that it is increasingly relevant in practice in industry and the future world of work.

\subsection{Discussion}

A possible explanation for the differences among the papers is that the achievement of HMS is not always a matter of "has" or "has not" but rather can be found along an implementation spectrum. Since none of the papers make this explicit, further research is needed to specify the number, characteristics, and granularity of what might be varying levels or degrees of HMS.

Nevertheless, some of the publications published in the last 15 years argue that HMS systems do already exist and express a positive attitude regarding. Studies of the boundaries and future of HMS show, however, that HMS systems are not always an optimal solution. It is therefore necessary to examine each individual possibility for implementation in the context of the HMS objective and conditions.

\section{Conclusion}

In this work, we have shown by means of a structured literature review that attention to HMS is increasing in the IS community. Aided by work from the last 60 years, we have created a first conceptualization for HMS. We worked out the basic concepts and requirements, have put them in relationship to each other, and have shown what the understanding of HMS looks like in the community. In doing so, we have helped establish a common vocabulary. From that common ground, our conceptualization can be used by IS scientists and practitioners who want to understand HMS.

\subsection{Implications}

Our conceptualization contributes to establishing some order to the many different facets of HMS in what is a very diverse literature, thus helping fulfill a need in IS research, where HMS is seen as an "emerging topic" [1]. Our conceptualization makes it possible to examine existing research from a different perspective and, for example, assess whether existing artifacts assigned to collaborative information systems already fulfill the requirements of HMS.

In addition, we have proposed a framework that describes how HMS systems are designed. It can be adapted, extended, and substantiated by IS researchers in the future.

Practitioners can use our work to understand how people and machines can work together in the future. We have given them a means to understand the concepts behind HMS and evaluate whether an HMS system is an option for how it will digitize in the future.

\subsection{Limitations and Further Research}

Section 6 discussed the future of HMS in detail. The discussion subsections above mention implications for further research. Here we present some further general research gaps.

To create a first conceptualization of HMS, our work focused closely on the term "symbiosis" and identified literature that uses that specific terms. We suspect that there is further work on collaborative systems in various fields that do not use the term but 
still implicitly fulfill all symbiosis requirements. Therefore, it would be interesting to follow up on our work with a broader examination of the literature on collaborative systems, with consideration of our conceptualization.

With respect to the term "machine," our work is at a more meta-level. We have raised Licklider's use of the term "computer" to a more general level to ensure our conceptualization would be independent of instantiations. In our literature search, we uncovered works that focus on the symbiosis between humans and robots, AI, vehicles, and instruments [7, 24, 35, 40]. It is conceivable that there are many other instantiations of HMS. Our inclusion of these four alone should not be seen as biasing our general conceptualization. For future work, it would be interesting to determine what other instantiations do exist, apply our conceptualization to them, and then generalize them again.

We have used our conceptualization to propose an initial framework for how HMS systems are designed. The proposal for the design framework in Section 5 is derived from the literature; we did not set out to prove it in this paper. We would like to encourage researchers to adapt, extend, and prove the framework with quantitative research or perhaps through case studies.

Based on having evaluated the HMS literature and creating our conceptualization, we do not see HMS as a new system type. Rather, HMS can rather be seen as a design philosophy and can be applied to a wide range of different system types in which collaboration could be improved through symbiosis. Further studies should deal with how HMS can be defined in the narrower sense.

Mutual benefit is emphasized as a fundamental goal of HMS. The literature identifies mutual learning as the only real mutual benefit in concrete terms. Future effort should be invested to investigate mutual benefit, especially with regard to the machine. Additional research also needs to focus on the benefit to the human.

Finally, we have not been able to identify any work that addresses the effects of human-machine symbiosis on working with systems or on their effectiveness. This is highly relevant, and should be taken up by the community.

\section{References}

[1] Abbass, H.A., G. Fogel, and J. Fidock, "Guest Editorial: Special Issue on Human-Machine Symbiosis", IEEE Transactions on Emerging Topics in Computational Intelligence 2(4), 2018, pp. 246-248.
[2] Adelé, S., and E. Brangier, "Evolutions in the Human Technology Relationship: Rejection, Acceptance and Technosymbiosis", IADIS International Journal on WWW/Internet 11(3), 2013, pp. 46-60.

[3] Anderson, W.T., "Augmentation, Symbiosis, Transcendence: Technology and the Future(s) of Human Identity", Futures 35(5), 2003, pp. 535-546.

[4] Arbib, M.A., "Artificial Intelligence: Cooperative Computation and Man-Machine Symbiosis", IEEE Transactions on Computers C-25(12), 1976, pp. 1346-1352. [5] Bary, A. de, Die Erscheinung der Symbiose: Vortrag, Gehalten auf der Versammlung Deutscher Naturforscher und Aerzte zu Cassel, Verlag von Karl J. Trübner, Strassburg, 1879.

[6] Bauer, L., "Compounds and Minor Word-Formation Types", In B. Aarts and A. McMahon, eds., The Handbook of English Linguistics. Blackwell Publishing, Malden, MA, USA, 2006, 483-506.

[7] Biondi, F., I. Alvarez, and K.-A. Jeong, "Human-Vehicle Cooperation in Automated Driving: A Multidisciplinary Review and Appraisal", International Journal of HumanComputer Interaction, 2019, pp. 1-15.

[8] Boff, K.R., "Revolutions and Shifting Paradigms in Human Factors \& Ergonomics", Applied Ergonomics 37(4), 2006, pp. 391-399.

[9] Brangier, É., and S. Hammes-Adelé, "Beyond the Technology Acceptance Model: Elements to Validate the Human-Technology Symbiosis Model", Proceedings of International Conference on Ergonomics and Health Aspects of Work with Computers, Springer Berlin Heidelberg (2011), $13-21$.

[10] Brynjolfsson, E., and A. McAfee, The Second Machine Age: Work, Progress, and Prosperity in a Time of Brilliant Technologies, W. W. Norton \& Company, 2014.

[11] Cesta, A., A. Orlandini, G. Bernardi, and A. Umbrico, "Towards a Planning-Based Framework for Symbiotic Human-Robot Collaboration", Proceedings of IEEE International Conference on Emerging Technologies and Factory Automation (ETFA), IEEE (2016), 1-8.

[12] Didakis, S., "The Myth of Symbiosis, Psychotropy and Transparency Within the Built Environment", Technoetic Arts: A Journal of Speculative Research 9(2-3), 2012, pp. 307-313.

[13] Döppner, D.A., D. Schoder, R.W. Gregory, and H. Siejka, "Exploring Design Principles for Human- Machine Symbiosis: Insights from Constructing an Air Transportation Logistics Artifact", Proceedings of International Conference on Information Systems (ICIS), (2016), 22.

[14] Eason, K.D., "Dialogue Design Implications of Task Allocation Between Man and Computer", Ergonomics 23(9), 1980, pp. 881-891.

[15] Ferreira, P., S. Doltsinis, and N. Lohse, "Symbiotic Assembly Systems - A New Paradigm", Procedia CIRP 17, 2014, pp. 26-31.

[16] Fitt, P.M., ed., Human engineering for an effective airnavigation and traffic-control system, Ohio State University Research Foudation, Washington, DC, USA, 1951.

[17] Gamberini, L., and A. Spagnolli, "Towards a Definition of Symbiotic Relations Between Humans and Machines", Symbiotic Interaction 2016, Springer International Publishing (2017), 1-4. 
[18] Griffith, D., "Neo-Symbiosis: A System Design Philosophy for Diversity and Enrichment", International Journal of Industrial Ergonomics 36(12), 2006, pp. 10751079.

[19] Haasbroek, L.J., "Advanced Human-Computer Interfaces and Intent Support: A Survey and Perspective", Proceedings of IEEE International Conference on Systems Man and Cybernetics Conference (SMC), IEEE (1993), 350 355.

[20] Hale, D.P., and G.M. Kasper, "The Effect of HumanComputer Interchange Protocol on Decision Performance", Journal of Management Information Systems 6(1), 1989, pp. $5-20$.

[21] Hollnagel, E., "Experimental Design of Man-Machine Studies", Social Science Information Studies 4(2-3), 1984, pp. 149-166.

[22] Hoßfeld, S., "Optimization on Decision Making Driven by Digitalization", Economics World 5(2), 2017.

[23] Jacucci, G., A. Spagnolli, J. Freeman, and L. Gamberini, "Symbiotic Interaction: A Critical Definition and Comparison to other Human-Computer Paradigms", Symbiotic Interaction 2014, Springer International Publishing (2014), 3-20.

[24] Jarrahi, M.H., "Artificial Intelligence and the Future of Work: Human-AI Symbiosis in Organizational Decision Making”, Business Horizons 61(4), 2018, pp. 577-586.

[25] Lesh, N., J. Marks, C. Rich, and C.L. Sidner, "ManComputer Symbiosis Revisited: Achieving Natural Communication and Collaboration with Computers", IEICE Transactions on Information and Systems 87-D(6), 2004, pp. 1290-1298.

[26] Lessiter, J., J. Freeman, A. Miotto, and E. Ferrari, "Ghosts in the Machines: Towards a Taxonomy of Human Computer Interaction", Symbiotic Interaction 2014, Springer International Publishing (2014), 21-31.

[27] Licklider, J.C.R., "Man-Computer Symbiosis", IRE Transactions on Human Factors in Electronics HFE-1(1), 1960, pp. 4-11.

[28] Mahler, M.S., On human symbiosis and the vicissitudes of individuation. Vol. 1: Infantile psychosis, Hogarth Press, London, 1969.

[29] Maier, M., A. Ebrahimzadeh, and M. Chowdhury, "The Tactile Internet: Automation or Augmentation of the Human?", IEEE Access 6, 2018, pp. 41607-41618.

[30] Manheim, M.L., "Issues in Design of Symbiotic DSS", Proceedings of the 22nd Hawaii International Conference on System Sciences (HICSS), IEEE (1989), 14-23.

[31] Mourad, S., and A. Tewfik, "Machine Assisted Human Decision Making", Proceedings of IEEE International Conference on Acoustics, Speech and Signal Processing (ICASSP), IEEE (2018), 6981-6985.

[32] Okoli, C., and K. Schabram, "A Guide to Conducting a Systematic Literature Review of Information Systems Research", Sprouts: Working Papers on Information Systems 10(26), 2010, pp. 1-51.

[33] Parker, L.E., and F.G. Pin, "Dynamic Task Allocation for a Man-Machine Symbiotic System", Proceedings of 1987 Goddard Conference on Space Applications of Artificial Intelligence (AI) and Robotics, (1987).
[34] Parsons, H.M., "The Scope of Human Factors in Computer-Based Data Processing Systems", Human Factors 12(2), 1970, pp. 165-175.

[35] Petriu, E.M., T.E. Whalen, I.J. Rudas, D.C. Petriu, and M.D. Cordea, "Human-Instrument Symbiotic Partnership for Multimodal Environment Perception", Proceedings of IEEE Instrumentation and Measurement Technology Conference (I ${ }^{2}$ MTC), IEEE (2008), 1263-1268.

[36] Pound, R., "Symbiosis and Mutualism", The American Naturalist 27(318), 1893, pp. 509-520.

[37] Ren, X., "Rethinking the Relationship between Humans and Computers", Computer 49(8), 2016, pp. 104-108.

[38] Saeed, A., M. Ammar, K.A. Harras, and E. Zegura, "Vision: The Case for Symbiosis in the Internet of Things", Proceedings of the 6th International Workshop on Mobile Cloud Computing and Services (MCS), ACM (2015), 23-27.

[39] Sanchez, J.C., and J.C. Principe, "Prerequesites for Symbiotic Brain-Machine Interfaces", Proceedings of IEEE International Conference on Systems, Man and Cybernetics (SMC), IEEE (2009), 1736-1741.

[40] Sandini, G., V. Mohan, A.M. Sciutti, and P. Morasso, "Social Cognition for Human-Robot Symbiosis-Challenges and Building Blocks", Frontiers in Neurorobotics 12(34), 2018, pp. 1-19.

[41] Sato, T., Y. Nishida, and H. Mizoguchi, "Robotic Room: Symbiosis with Human Through Behavior Media", Robotics and Autonomous Systems 18(1-2), 1996, pp. 185-194.

[42] Scriabin, M., D.B. Kotak, and K.G. Whale, "Symbiotic Systems: Exploiting Human Creativity", European Journal of Operational Research 84(2), 1995, pp. 227-234.

[43] Sheth, A., P. Anantharam, and C. Henson, "Semantic, Cognitive, and Perceptual Computing: Paradigms That Shape Human Experience", Computer 49(3), 2016, pp. 64-72.

[44] Spagnolli, A., M. Conti, G. Guerra, J. Freeman, D. Kirsh, and A. van Wynsberghe, "Adapting the System to Users Based on Implicit Data: Ethical Risks and Possible Solutions", Symbiotic Interaction 2016, Springer International Publishing (2017), 5-22.

[45] Sun, R., "Potential of Full Human-Machine Symbiosis Through Truly Intelligent Cognitive Systems", AI \& Society, 2017, pp. 1-12.

[46] Webster, J., and R.T. Watson, "Analyzing the Past to Prepare for the Future: Writing a Literature Review", MIS Quarterly 26(2), 2002, pp. xiii-xxiii.

[47] Wolfswinkel, J.F., E. Furtmueller, and C. Wilderom, "Using Grounded Theory as a Method for Rigorously Reviewing Literature", European Journal of Information Systems 22, 2013, pp. 45-55.

[48] Xu, Z., H. Liao, B. Li, and W. Li, "Vega Grid and CSCW: Two Approaches to Collaborative Computing", Proceedings of the 8th International Conference on Computer Supported Cooperative Work in Design (CSCWD), IEEE (2004), 10-17.

[49] ISO 9241-210:2010: Ergonomics of Human-System Interaction -- Part 210: Human-Centred Design for Interactive Systems, ISO, Switzerland, 2010.

[50] "machine, n.", OED Online, 2019. www.oed.com/view/Entry/111850 